\title{
Haltungsasymmetrie bei Säuglingen: Beobachtung, Handling und Lagerung
}

Kai Stoevesandt

Bei Säuglingen, die viel auf dem Rücken gelagert werden, zeigen sich häufig Kopfverformungen und Haltungsasymmetrien. Diese können gravierende Folgen haben. Als Physiotherapeut und Osteopath möchte ich Sie mit diesem Artikel und mit meinem Vortrag auf dem FORUM HEBAMMENARBEIT in Wiesbaden dabei unterstützen, die Symptome zu erkennen, die Hintergründe zu verstehen und adäquate Maßnahmen zu ergreifen. Denn gerade Hebammen können in den ersten Lebensmonaten einen wesentlichen Beitrag zur Prävention leisten.

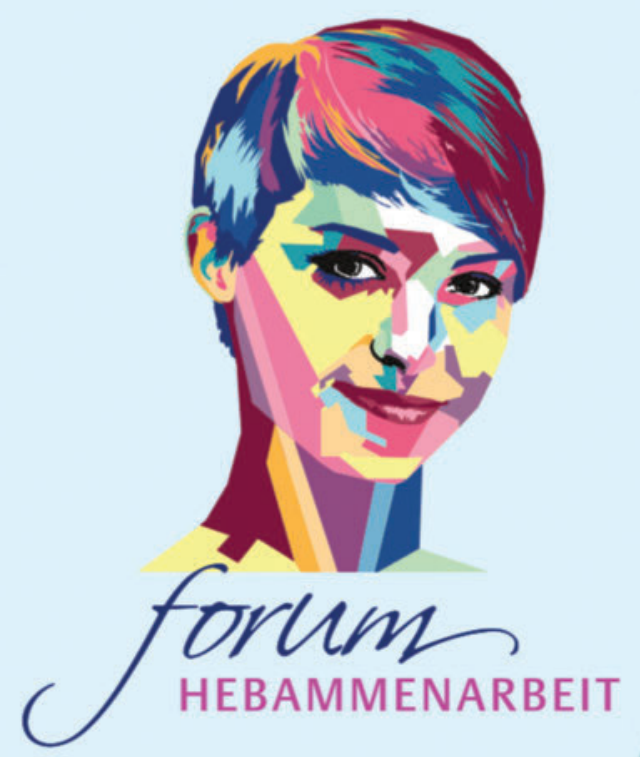

Erleben Sie den Vortrag von Kai Stoevesandt live beim Forum Hebammenarbeit vom 8. -9.11.2019 in Wiesbaden.

\section{Medizinischer Hintergrund}

Eine Kopfverformung kommt relativ häufig vor und betrifft rund 70.000 Säuglinge in Deutschland. Bei etwa einem Viertel davon entwickelt sich daraus eine skoliotische Fehlhaltung, d.h. eine Haltungsasymmetrie mit weiteren negativen Folgen. Säuglinge sind in ihren ersten 8-12 Lebenswochen besonders davon betroffen, weil ihr Schädel noch sehr schnell wächst und verformbar ist [2]. Liegt der Säugling viel auf dem Rücken, wie es als Prävention gegen den Plötzlichen Kindstod empfohlen wird, oder hat er eine Lieblingsseite, zu der er den Kopf überwiegend dreht, so kommt es aufgrund des Eigengewichts des Schädels zu einer Kopfasymmetrie, einem sogenannten lagebedingten Plagiocephalus. Da 
seit 1991 die Empfehlung gilt, Säuglinge konsequent auf dem Rücken schlafenzulegen, ist zwar die Häufigkeit des Plötzlichen Kindstods von über 1.200 Fällen pro Jahr auf unter 200 gesunken [5], die Anzahl der Säuglinge mit lagebedingtem Schiefschädel ist jedoch gleichzeitig signifikant angestiegen [3]. Das hängt auch damit zusammen, dass der Säugling seinen Kopf nicht willentlich anders positionieren kann, wenn dieser an einer Seite abgeflacht ist. Aufgrund fehlender motorischer Reife, aber auch allein aus physikalischen Gründen rollt der Hinterkopf immer wieder in dieselbe Position [10].

\section{Mögliche Folgen der Kopfverformung}

Die Kopfverformung wirkt sich zunächst auf die Schädelbasis und somit auf die Ohrachse aus und beeinträchtigt den Gleichgewichtssinn ( $\triangleright$ Abb.1). Werden die Kiefergelenke verschoben, kann ein Kreuzbiss entstehen oder die Zahnokklusion betroffen sein ( Abb. 2) [6][7].

Des Weiteren verändert die Kopfverformung die Strukturen der Schädelbasis mit den Halswirbeln und den dortigen Nervenbahnen [11]. Eine Fehlspannung im Bereich der oberen Halswirbelsäule und der Kopfgelenke beeinträchtigt die dortige muskuläre Struktur. Es kann ein sogenannter Schiefhals, aber auch eine Fehlstellung der Wirbelsäule entstehen [4][14]. Als Teil der Schädelbasis kann sich die Veränderung auch auf die Pars condylaris

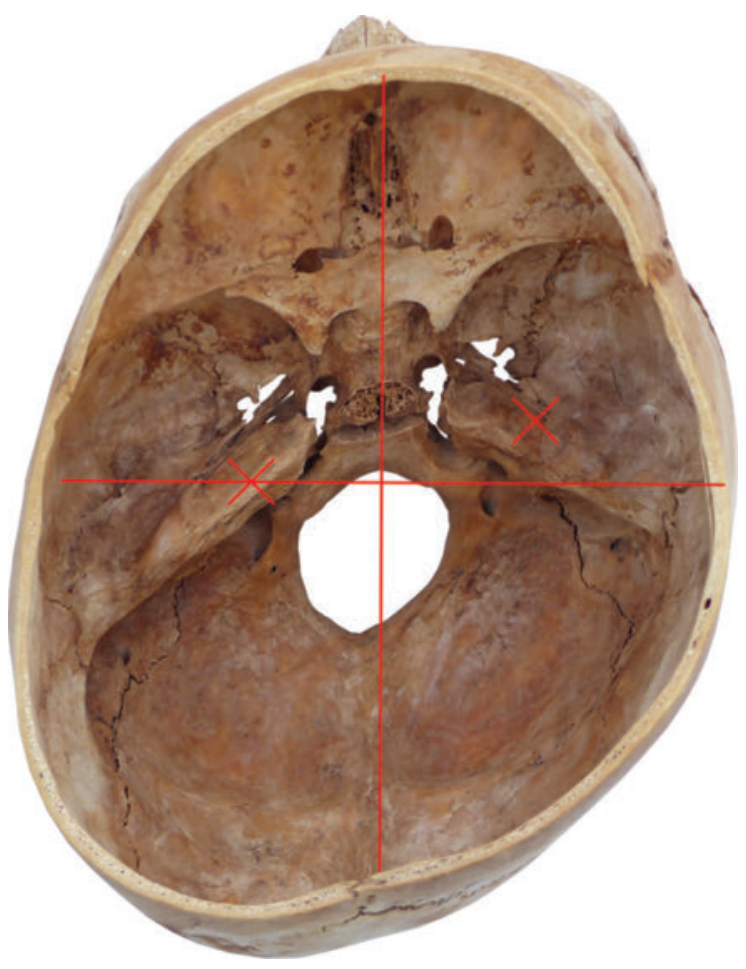

- Abb. 1 Schädelbasis von oben mit verschobenen Gleichgewichtsorganen durch Plagiocephalus. (Quelle: Kai Stoevesandt) des Os occipitale und damit auf das Foramen jugulare auswirken. Hier verlaufen wichtige Nervenstrukturen. Sie können durch eine Fehllagerung, aber auch schon während des Geburtsvorgangs komprimiert werden, wenn sich die Strukturen der weichen Schädelbasis durch den Druck der Gebärmutter verschieben. Von solchen strukturellen Veränderungen können Funktionen wie Stillen bzw. Schlucken und Trinken betroffen sein.

\section{Haltungsasymmetrie}

Bilden Säuglinge eine Haltungsasymmetrie aus, können sich sensorische Beeinträchtigungen ergeben [4][14]. Das hängt mit den Kopfgelenkstrukturen zusammen: Die dortigen Muskeln bilden die sog. Nackenrosette. Sie sind mit sehr vielen Fühlermechanismen ausgestattet, um das Gehirn darüber zu informieren, wie sich Kopf und Rumpf zueinander verhalten. Sie stehen in Verbindung mit dem Gleichgewichtssinn, der jedoch bei einer Verschiebung der Ohrachse keine korrekten Informationen mehr an das Gehirn liefern kann. Dann vertikalisiert sich das Kind nicht mehr so, dass es lotrecht aufgerichtet sitzt oder steht, und es entwickelt eine skoliotische Fehlhaltung. Je länger dieser Zustand anhält, desto stärker bilden sich die entsprechenden Nervenbahnen aus, wodurch ein Umpolen der nervalen Verbindungen, z.B. durch korrigierende Reize in die korrekte Haltungsposition umso langwieriger wird. Die mit einer Haltungsasymmetrie assoziierten Bewegungsmuster stimulieren demnach die Synapsenbildung einseitig.

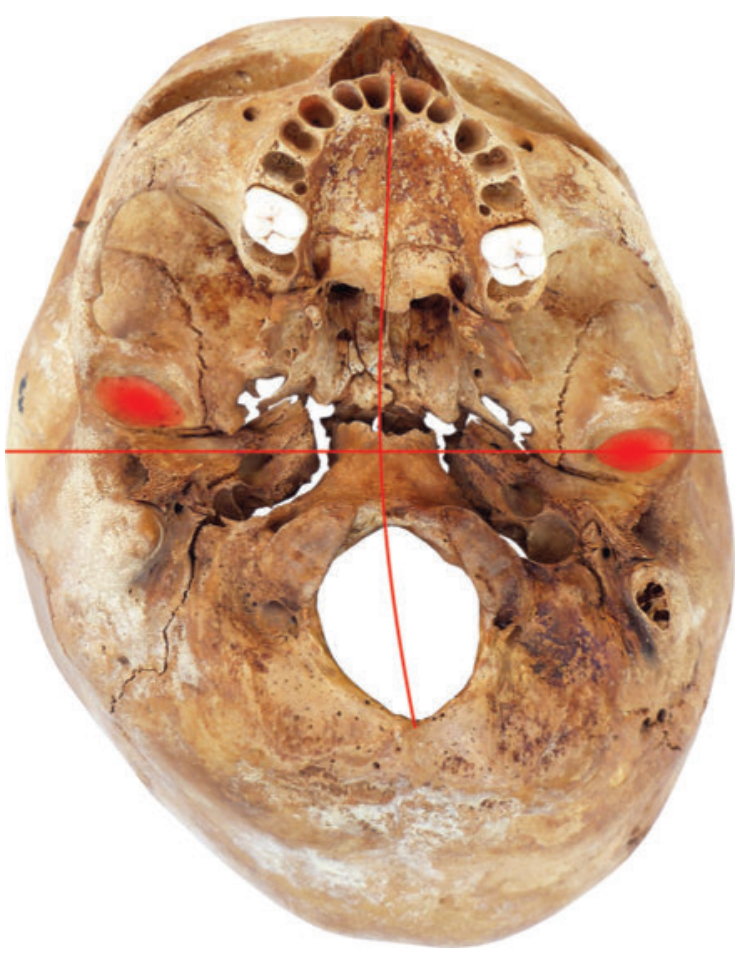

Abb. 2 Schädelbasis von unten mit verschobenen Kiefergelenken durch Plagiocephalus. (Quelle: Kai Stoevesandt) 
Merke

Hebammen können bei Säuglingen mit einer Haltungsasymmetrie durch die Vermittlung von steuerndem Handling, Bauchlagentraining und durch Empfehlung geeigneter Therapieformen die motorische Verhaltensänderung sowie die Synaptogenese positiv beeinflussen.

Es gibt aber auch andere Gründe für eine Haltungsasymmetrie, wie z.B. eine Stauchung der Schädelbasis beim Geburtsvorgang oder invariante/s Lagerung/ Handling. Ein Auge des Kindes kann dominanter sein oder ein Ohr schlechter hören, was wiederum eine Fehlhaltung begünstigt, da das bessere Organ stärker in den Raum gestellt wird. Ebenso sind Erkrankungen des Zentralnervensystems sowie Gelenkstörungen der Halswirbelsäule möglich. Eine Fraktur der Klavikula sowie eine ArmPlexusparese können zu einer Schonhaltung führen und der Ausbildung einer Haltungsasymmetrie Vorschub leisten.

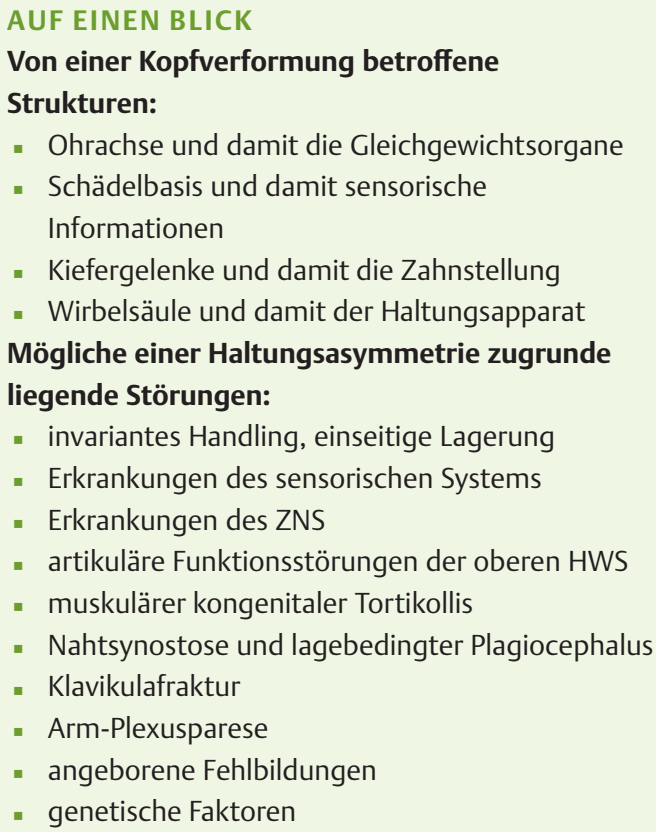

Je nach Ausprägung und Schweregrad sollten verschiedene Fachkräfte involviert werden [8]. Für die Behandlung der Informationsstörung der Nervenbahnen ist ein Arzt, für die Fehlstellung der Kopfgelenke ein Orthopäde oder ein Physiotherapeut gefragt.
Hier würden Sie als Hebamme den Eltern einen großen Dienst erweisen, wenn Sie sie in dieser Beobachtung schulen und ihnen Gegenmaßnahmen vermitteln. Denn Ihr Kontakt zu den Familien ist insbesondere in den ersten Lebenswochen der Säuglinge viel engmaschiger als der des Kinderarztes.

\section{Früherkennung einer Haltungsasymmetrie}

Ausgangspunkt für die Früherkennung ist die Beurteilung der Wirbelsäule und der Kopfform. Als erstes wird überprüft, ob sich das Baby gegen die Schwerkraft aufrichten kann. Hierfür wird es hochgehoben und zur Seite gekippt. Ein gesundes Baby richtet sich selbst gegen die Schwerkraft auf oder hält sich mindestens gerade ( $\triangleright$ Abb.3). Hängt es hierbei jedoch durch, lässt sich eine einseitige Schwäche der Rumpfmuskulatur erkennen ( $\triangleright$ Abb.4).

Als zweite Maßnahme wird getestet, ob das Baby den Kopf um 180 Grad drehen kann (>Abb.5). Liegt das Baby auf dem Rücken und wird es mithilfe eines Gegenstands dazu angeregt, zur Seite zu blicken, sollte sich seine Wirbelsäule gleichzeitig auf der Gesichtsseite lang, also konvex, einstellen. Man spricht hier von einem reaktiven Bewegungsmoment der Wirbelsäule bei zervikaler Rotationsfähigkeit. Das heißt, dreht das Kind den Kopf in der Rückenlage nach rechts, biegt sich die Wirbelsäule ebenfalls konvex nach rechts. Blickt das Kind hingegen in Bauchlage nach rechts, öffnet sich die Wirbelsäule konvex nach links, also auf der Hinterhauptseite.

Mit diesem Test lässt sich erkennen, ob das Kind seinen Kopf frei rotieren kann und ob sich die Wirbelsäule physiologisch korrekt einstellt. Falls nicht, sollte als nächstes der M. sternoclaidomastoideus (großer KopfwenderMuskel) abgetastet werden. Bei einer Fehlspannung kann sich hier eine knotenartige Verhärtung in der Größe einer Bohne tasten lassen. Oder der Muskel verhärtet sich und entwickelt innerhalb der ersten acht Lebenswochen des Säuglings eine Strangbildung. Dann wäre eine weitere Untersuchung der muskulären Strukturen der Halswirbelsäule sowie der artikulären Strukturen der Kopfgelenke durch einen Arzt oder einen Therapeuten angeraten.

Für eine Beurteilung der Kopfform ist die Vogelperspektive einzunehmen ( $\triangleright$ Abb.6). Von hier aus lässt sich erkennen, ob die Ohrachse verschoben ist.

- Wenn eine Hinterhauptseite abgeflacht ist und dabei das Ohr auf dieser Seite zur Stirn verschoben ist, handelt es sich um einen lagebedingten Plagiocephalus.

- Ist das Ohr auf der Schädelseite, die abgeflacht ist, jedoch nach hinten gezogen, liegt eine vorzeitige Nahtsynostose vor. 


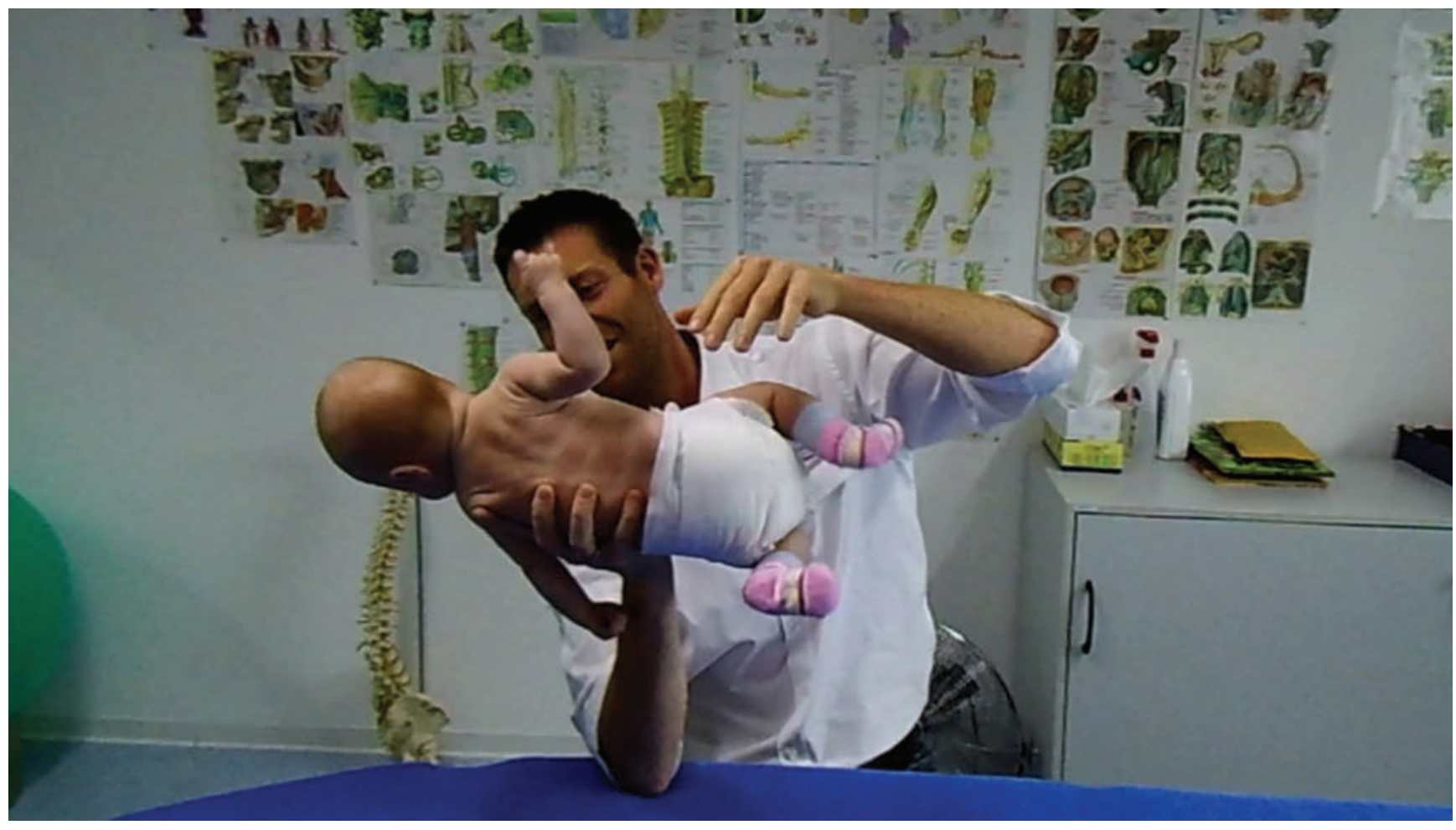

Abb. 3 Seitkippreaktion bei kräftiger Rumpfmuskulatur - das Baby richtet sich auf bzw. hält sich gerade. (Quelle: Kai Stoevesandt [rerif])

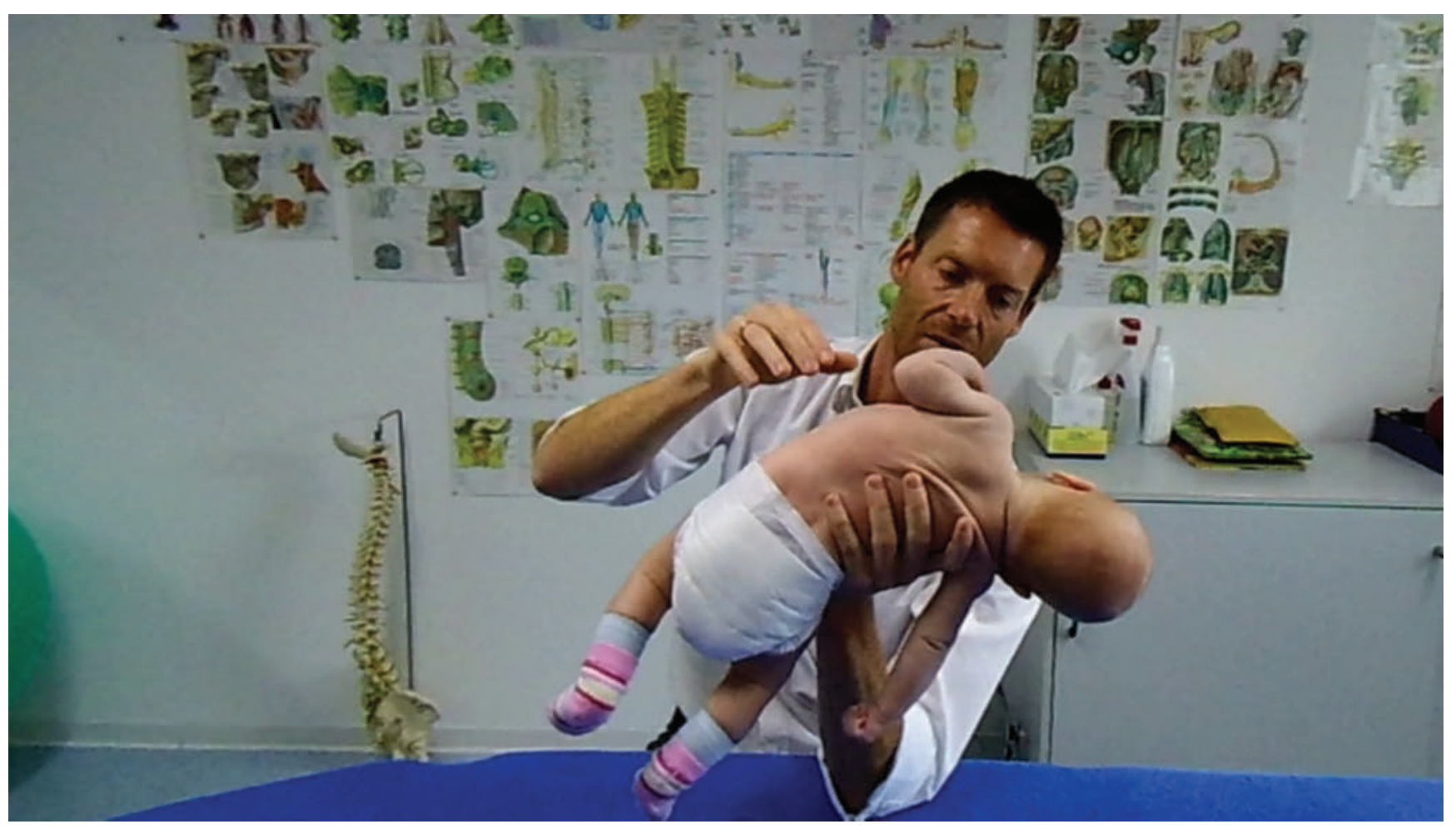

Abb. 4 Seitkippreaktion bei einseitig schwacher Rumpfmuskulatur - das Baby ist nicht in der Lage, sich gegen die Schwerkraft aufzurichten. (Quelle: Kai Stoevesandt [rerif]) 


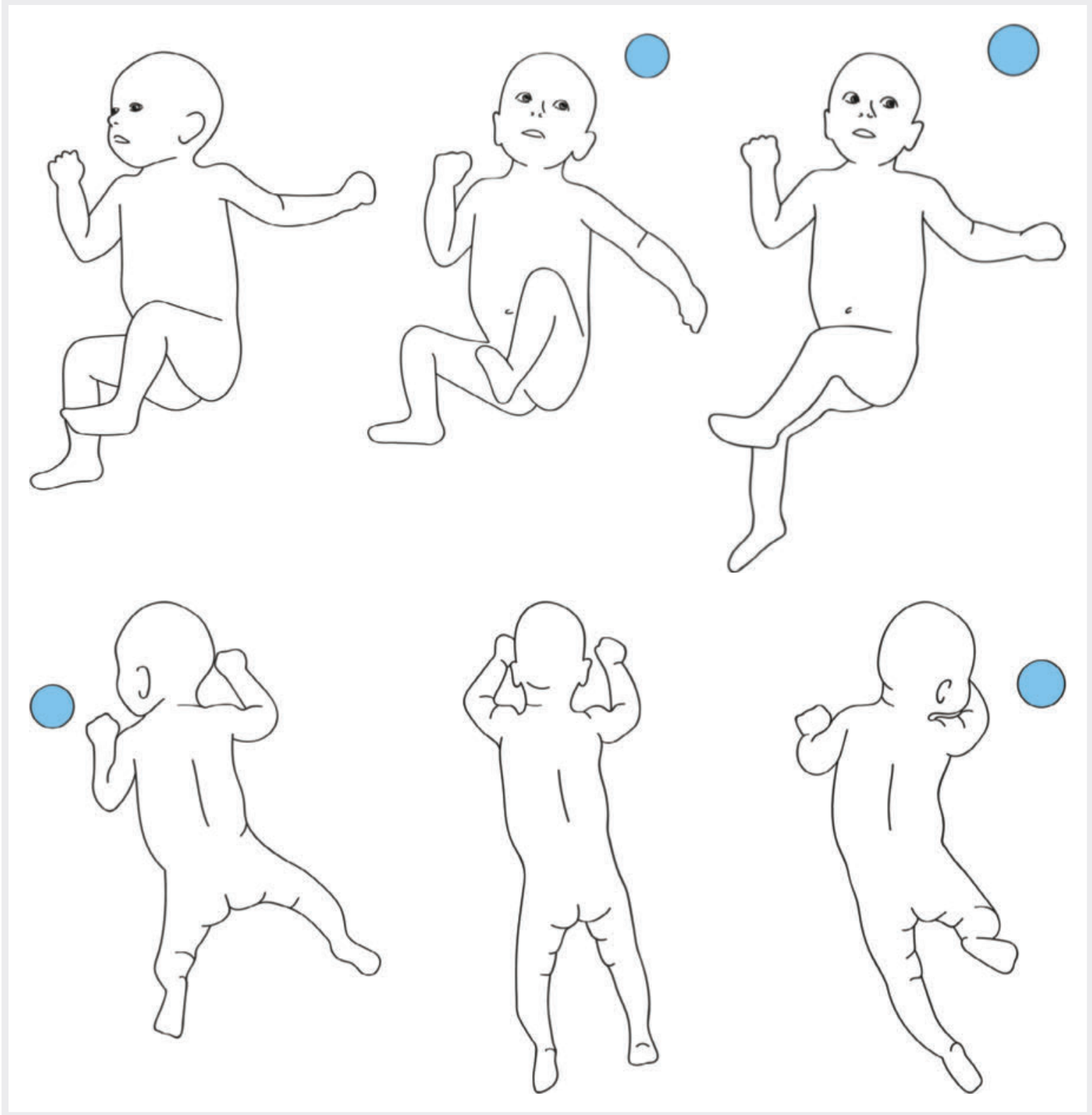

Abb. 5 Test der reaktiven Haltungsbewertung in Rücken- und Bauchlage. Ohne Befund: Die orientierende Kopfdrehung ist in Rücken- wie Bauchlage frei. In Rückenlage stellt sich dabei die Wirbelsäule auf der Gesichtsseite konvex ein. In Bauchlage öffnet sich die Wirbelsäule hingegen konvex auf der Hinterhauptsseite. Befundbeispiel - Abbildung: Eingeschränkte cervikale Rotation nach links in Rückenlage. In Bauchlage mit Kopfdrehung links biegt sich die Wirbelsäule nicht nach rechts konvex. (Quelle: Kai Stoevesandt)

\section{Maßnahmen und Empfehlungen für die Eltern}

Wenn Sie eine lagerungsbedingte Kopfverformung erkennen, ist es wichtig, die Eltern aktiv in die Behandlung einzubeziehen. Zunächst können die Eltern lernen, die Kopfform ihres Babys zu beurteilen und die Haltungsasymmetrie wahrzunehmen [12]. Hieran erkennen sie auch, wie dringend der Handlungsbedarf ist.

Wenn das Baby wach ist, sollte fünfmal am Tag für jeweils $5 \mathrm{~min}$ ein Bauchlagentraining durchgeführt werden [7][10]. Hiermit lassen sich einerseits die motorischen Kompetenzen des Kindes fördern, andererseits kann damit die Vorzugshaltung gezielt ausgeglichen werden. Dafür wird der Säugling in Bauchlage angeregt, zu der Seite zu gucken, die ihm (noch) nicht so sehr behagt. Um den Blick zu lenken, kann die Hand als Scheuklappe dienen. Auch ein Spielzeug, ein Glöckchen oder Ähnliches können als Reiz eingesetzt werden.

Auch das Tragen sollten die Eltern jetzt bewusster vornehmen. Dafür empfiehlt sich das sogenannte Handling nach Bobath ( $\triangleright$ Abb.8, $\triangleright$ Abb.9). Dabei sollte das 


\section{INFO}

\section{Erkennungsmerkmale und Untersuchungsmethoden}

Folgende Merkmale deuten auf eine Haltungsasymmetrie bei Säuglingen hin:

- Beobachtung einer Positionspräferenz

- Asymmetrische Bewegungsmuster im Rahmen der reaktiven Haltungsbewertung

- Eingeschränkte Halswirbelsäulenbeweglichkeit

- Positiver Tastbefund des M. sternoclaidomastoideus

- Schiefe Kopfhaltung im Traktionsversuch

- Mangelnde einseitige Aufrichtungskompetenz in der Seitkippreaktion

- Beurteilung der Kopfform (Plagiocephalometry)

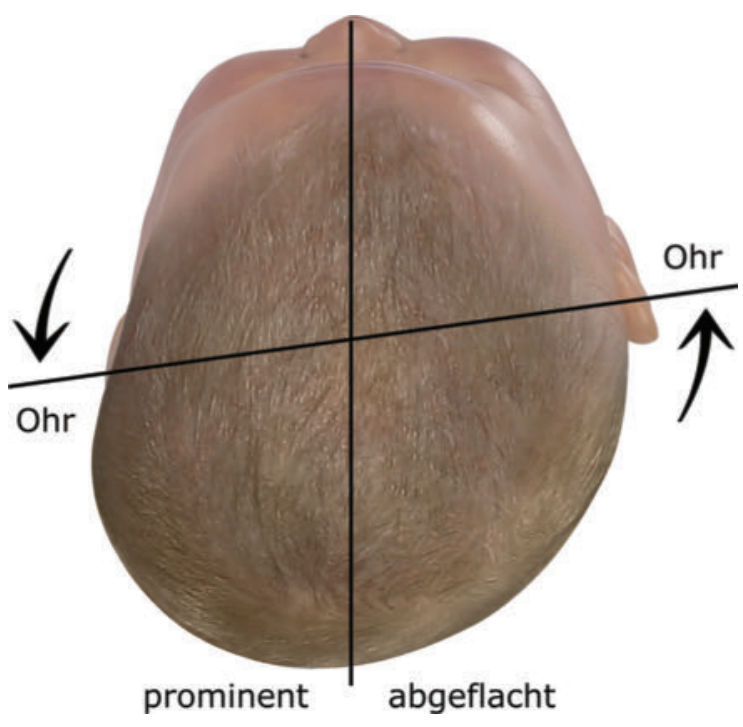

Abb. 6 Beurteilung der Kopfform aus der Vogelperspektive. (Quelle: Kai Stoevesandt)

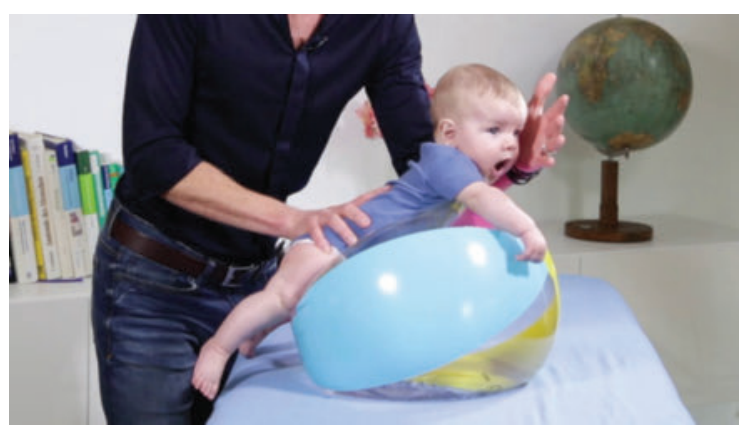

Abb. 7 Mit Bauchlagenübungen auf dem Wasserball trainiert das Baby, die weniger präferierte Seite zu fokussieren. (Quelle: Kai Stoevesandt [rerif])
Baby immer so gehalten werden, dass es in die Richtung schauen muss, die es aufgrund der vorherrschenden Positionspräferenz selbst nicht wählen würde. Wenn also bisher die linke Rumpfseite schwach ausgebildet ist und das Baby in der rechten Armbeuge von Mutter oder Vater getragen wird, muss es sich aus eigener Kraft nach links gegen die Schwerkraft aufrichten und trainiert so die bisher vernachlässigten Muskeln und Nervenbahnen.

Da die Schlafenszeit noch verhältnismäßig lang ist, liegt hier eine große Chance für die Behandlung der Kopfverformung: So wie sich der Schädel verformt hat, kann er sich auch wieder zurückbilden-sofern der Kopf richtig gelagert wird. Hier spricht man dann von einer Lagerungstherapie [2][10]. Für die gewünschte Wachstumslenkung muss das Kind seitlich so zum Schlafen gelegt werden, dass sein Köpfchen auf der Seite des prominenten Hinterhaupts ruht (Abb. 10).

Bis zur 12. Lebenswoche reicht es durchaus, das Baby hierfür in einer Zwischenposition zwischen Rücken- und Seitenlage mit Handtuchrollen im Rücken und am Bauch zu sichern [5]. Ab der 13. Lebenswoche ist dies jedoch nicht mehr zulässig und darf auch nicht mehr empfohlen werden, da der Säugling jetzt motorisch so reif und auch so stark ist, dass er sich von allein in die Rücken- oder Bauchlage zu drehen beginnt. Die Rückenlage ist wegen des Plagiocephalus ungünstig, die unbeobachtete Bauchlage wegen des Plötzlichen Kindstods sogar gefährlich. Doch eine seitliche Lagerung-bereits in den frühen Lebenswochen-ist eine gute Prävention, wenn sie variabel vorgenommen wird, sodass das Kind kaum mehr eine einseitige Präferenz ausbilden wird [1].

Bei einer Kopfverformung sollten Sie eine abgesicherte Lagerung empfehlen, mit deren Hilfe das Kind im Schlaf so mit den unterstützenden Kissen verbunden ist, dass diese nicht wegrutschen können und der Säugling somit keinen Halt mehr hätte [13][10]. Diese Bedingungen kann eine Seitenlagerungsschiene erfüllen, die zur Vermeidung bzw. Korrektur lagebedingter Kopfverformungen bei Säuglingen im 1. Lebensjahr geeignet ist [10]. Die Wirksamkeit der Lagerungstherapie wurde in Studien gezeigt [9].

\section{TIPP}

Empfehlungen für die Eltern

- Bauchlagentraining $5 \times 5 \mathrm{~min} / \mathrm{Tag}$

- Handling nach Bobath

- spezifische Übungen

- seitliche Lagerung (70\% der Liegezeit)

Wird ein Säugling mit Plagiocephalus zu $70 \%$ seiner Liegezeit auf die prominente Hinterhauptseite gelegt, 


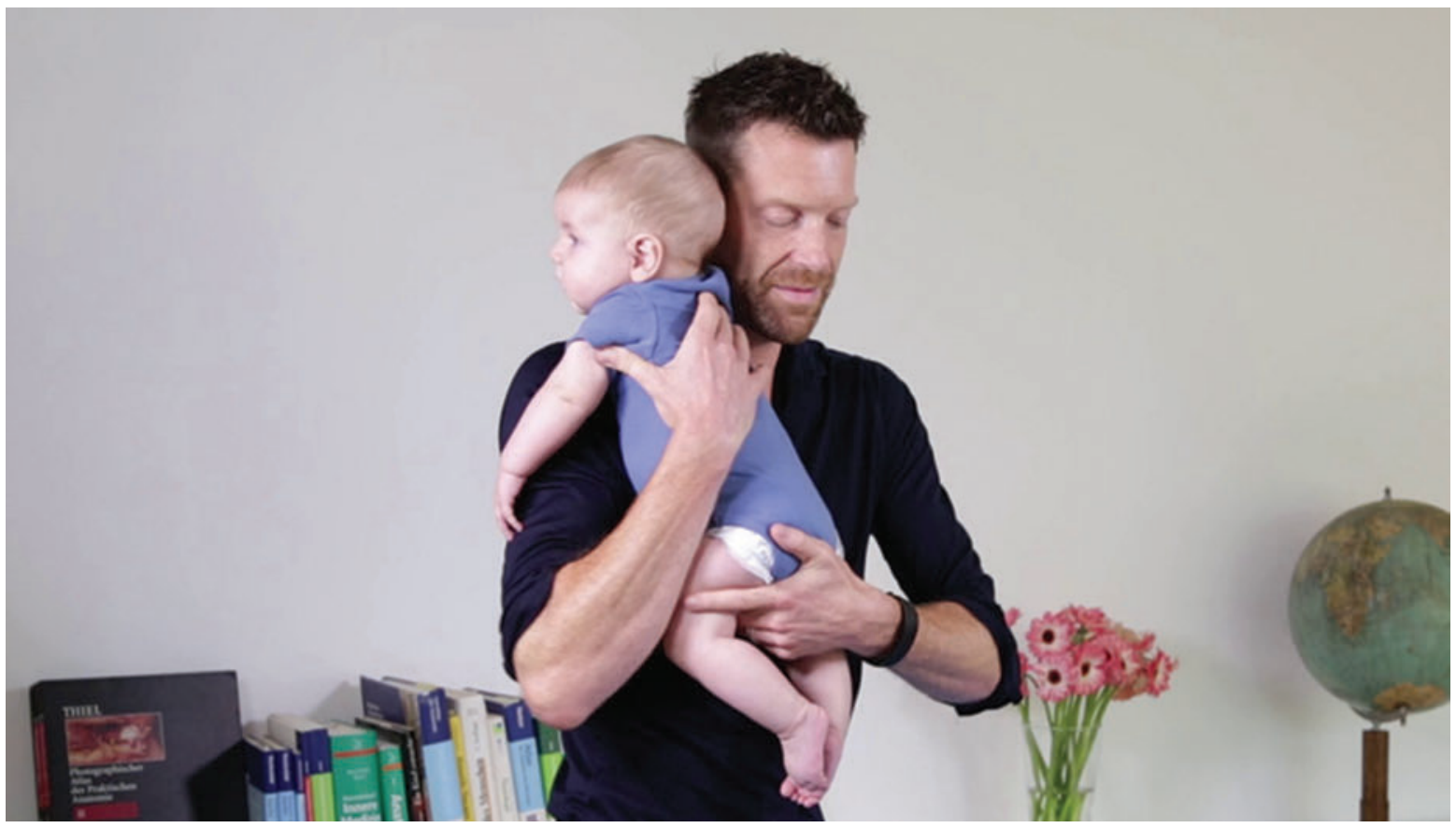

Abb. 8 Handling nach Bobath: Kopfrotation entgegen der präferierten Seite. (Quelle: Kai Stoevesandt [rerif])

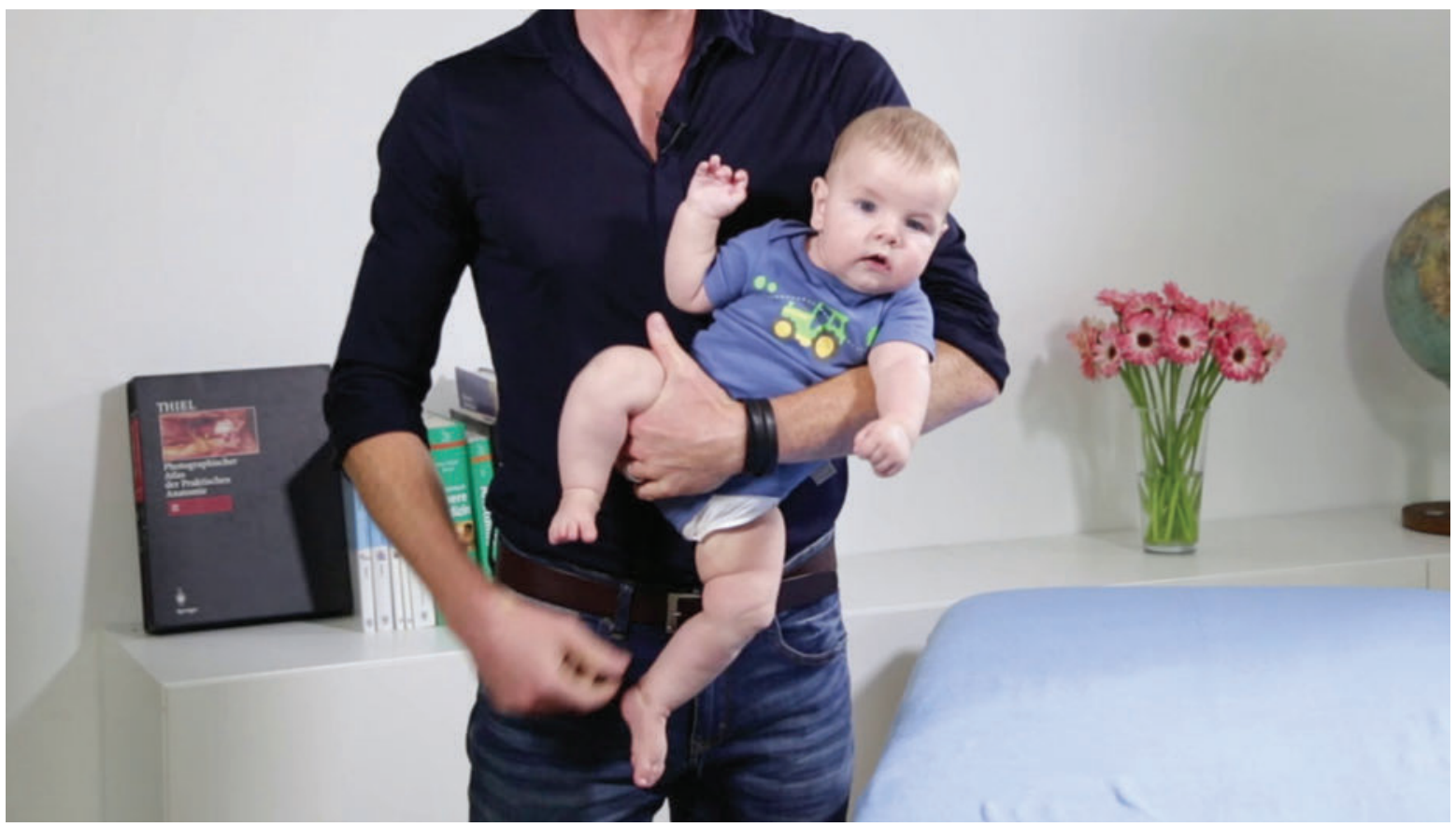

Abb. 9 Handling nach Bobath: Das Baby wird so getragen, dass es sich seitlich gegen die Schwerkraft aufrichten muss. (Quelle: Kai Stoevesandt [rerif])

so zeigen die Erfahrungswerte sehr positive Ergebnisse: Die Diagonalendifferenz der horizontalen Schädeldiagonalen kann zwischen dem 4 . und 6 . Lebensmonat um $1 \mathrm{~mm}$ pro Woche reduziert werden ( A Abb 10). In der Zeit bis zum 9. Lebensmonat dauert es etwa 10 Tage, um diese Korrektur zu erreichen [2][10].
Indem der Kopf wieder seine symmetrische Form erhält, kommen die Gleichgewichtsorgane wieder ins Lot und die Kiefergelenke passen wieder korrekt zueinander. Der Kopf und die Wirbelsäule richten sich gerade aus, die Muskel- und die Nervenbahnen entwickeln sich gleichmäßig und das Gehirn erhält die korrekten Informationen über die Bezüge der Organe und Gliedmaßen zueinander. 


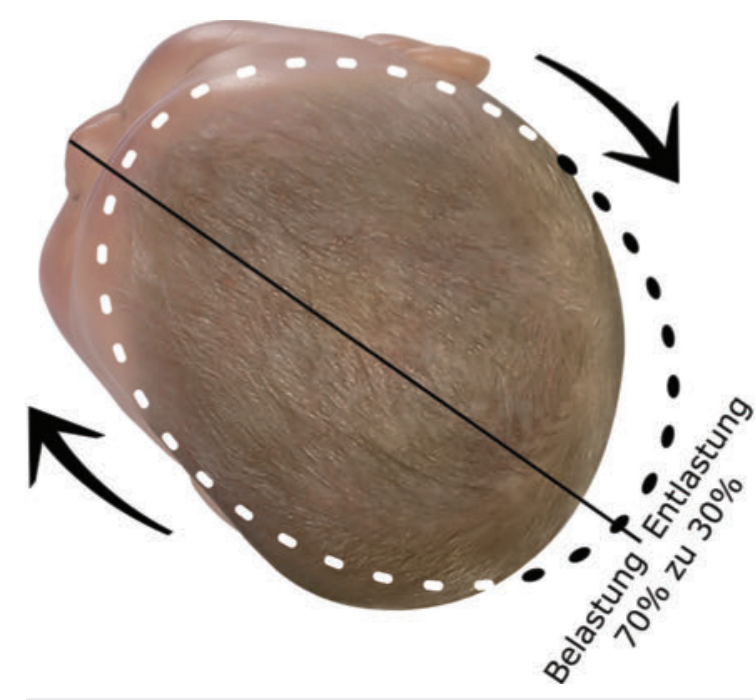

- Abb.10 Lagerung auf der Seite des prominenteren Hinterhaupts zur Wachstumslenkung während der Schlafenszeit. (Quelle: Kai Stoevesandt)

\section{AUSBLICK}

Der vorliegende Artikel über den Umgang mit Säuglingen, die eine Haltungsasymmetrie und eine lagebedingte Kopfverformung erkennen lassen, möchte die Dimensionen aufzeigen, die ein

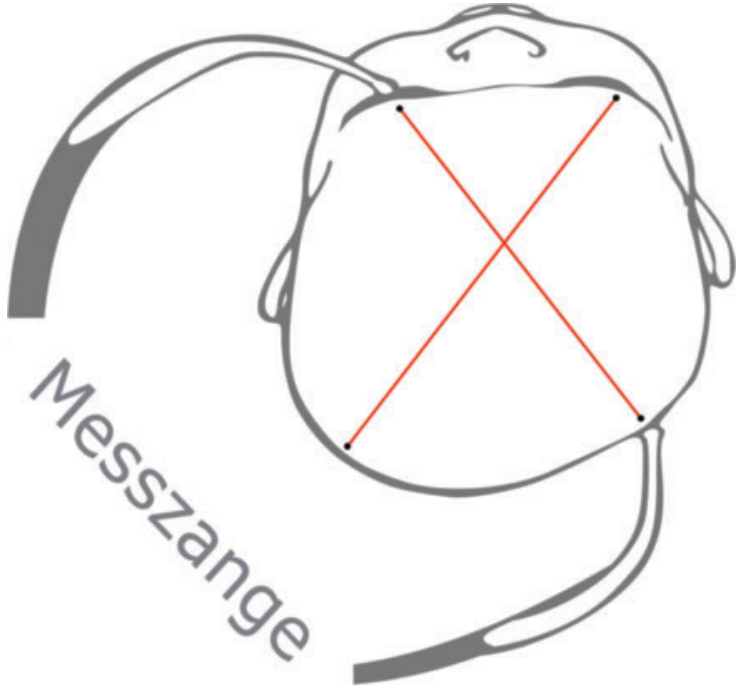

- Abb.11 Durch gezielte Maßnahmen lässt sich die Differenz der horizontalen Schädeldiagonalen in den ersten Lebensmonaten messbar reduzieren. (Quelle: Kai Stoevesandt)

solcher, scheinbar äußerlicher Prozess, nach sich ziehen kann. Dabei können Sie als Hebamme mit geschultem Blick präventiv einen wertvollen Beitrag leisten. Mit Ihrer Unterstützung lernen die Eltern selbst, ihr Baby aufmerksam zu begleiten 
und es im Falle einer einseitigen Entwicklung aktiv zu fördern bzw. richtig zu lagern. Da dies in Deutschland statistisch gesehen rund 70.000 Babys pro Jahr betrifft, von denen ca. ein Viertel eine Haltungsasymmetrie ausbildet, lässt sich gut erkennen, wie nachhaltig hier eine frühzeitige Begleitung von Eltern und Babys sein kann.

\section{Über diesen Artikel}

Dieser Artikel fasst den Vortrag von Kai Stoevesandt auf dem Forum Hebammenarbeit 2019 zusammen. Der Autor referiert zum Thema „Haltungsasymmetrien bei Säuglingen“ am 9. November von 15.30 bis 16.30 im RheinMain CongressCenter Wiesbaden. Programm und Anmeldung unter: www.forumhebammenarbeit.de Weitere Informationen über Haltungsasymmetrie und die Auswirkungen einer Blockade der Kopfgelenke gibt der Autor auf folgender Webseite: www.varilag.de/ratgeber/ fachinformationen

\section{Autorinnen / Autoren}

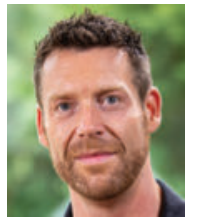

Kai Stoevesandt ist Dipl. Physiotherapeut (FH) Osteopath und Fachbereichsleiter Physiotherapie am Ambulanten Zentrum für Rehabilitation und Prävention am Entenfang GmbH (AZR) in Karlsruhe.

\section{Interessenkonflikt}

Zu Kai Stoevesandts Schwerpunkten als Physiotherapeut zählt die Behandlung von Haltungsasymmetrien bei Säuglingen. Der Autor ist Inhaber von Varilag ${ }^{\circledR}$ und vertreibt das von ihm entwickelte Lagerungskissen zur Vermeidung bzw. Korrektur lagebedingter Kopfverformungen bei Säuglingen.

\section{Korrespondenzadresse}

Kai Stoevesandt

Ambulantes Zentrum für Rehabilitation und Prävention am Entenfang $\mathrm{GmbH}$

Am Entenfang 12-14

76185 Karlsruhe

E-Mail: k.stoevesandt@azr.de

\section{Literatur}

[1] Aarnivala HE, Vuollo V, Harila V et al. Preventing deformational plagiocephaly through parent guidance: a randomized, controlled trial. EurJPediatr 2015; 174(9):1197-1208

[2] Aarnivala HE, Vuollo V, Harila V et al. The course of positiona cranial deformation from 3 to 12 months of age and associated risk factors: a follow-up with 3D imaging. EurJPediatr 2016; 175(12):1893-1903

[3] De Bock F, Braun V, Renz-Polster H. Deformational plagiocephaly in normal infants: a systematic review of causes and hypotheses. ArchDisChild 2017; 102(6):535-542

[4] Feijen M, Franssen B, Vincken $\mathrm{N}$ et al. Prevalence and consequences of positional plagiocephaly and brachycephaly. J Craniofac Surg 2015; 26(8):770- 773

[5] Jorch G. Prävention des Plötzlichen Kindstodes. Monatsschr Kinderheilkd 2010; 158: 564-569

[6] Kluba S, Rosskopf F, Kraut W et al. Maloclusion in the primary dentition in children with and without deformational plagiocephaly. Clin Oral Investing 2016; 20: 2395-2401

[7] Linz C, Kunz F, Böhm $\mathrm{H}$ et al. Positional skull deformitiesetiology, prevention, diagnosis, and treatment. DtschArtzebIInt 2017; 114: 535-542

[8] Richter D. Physiotherapie bei lagerungsbedingter Plagiozephalie. Zeitschrift für Physiotherapeuten 2017: 51-52

[9] Steinberg JP, Rawlani R, Humphries LS, Rawlani V, Vicari FA: Effectiveness of conservative therapy and helmet therapy for positional cranial deformation. Plast Reconstr Surg 2015; 135 : $833-42$

[10] Stoevesandt K, Ma H, Beyer U et al. Lagerungsplagiozephalus beim Säugling. Epidemiologie, Pathophysiologie, Prophylaxe, Diagnostik und Therapieoptionen. Monatschr Kinderheilkd 2018; 166(8):675-682

[11] Stücker R. Die mit Plagiozephalus assoziierte Säuglingsasymmetrie. Z Orthop Unfall 2009; 147: 503-512

[12] van Vlimmeren LA, van der Graaf Y, Boere-Boonekamp MM et al. Effect of pediatric physical therapy on deformational plagiocephaly in children with positional preference: a randomized controlled trial. Arch Pediatr Adolesc Med 2008; 162 (8): $712-718$

[13] Wilbrand JF, Seidl M, Wilbrand M et al. A prospective randomized trial on preventative methods for positional head deformity: physiotherapy versus a positioning pillow. J Pediatr 2013; 162(6):1216-1221

[14] Zhao XQ, Wang LY, Zhao CM et al. Neurological assessment of Chinese infants with positional plagiocephaly using a Chinese version of the Infant Neurological International Battery (INFANIB). ChildsNervSyst 2017; 33(2):281-288

\section{Bibliografie}

DOI https://doi.org/10.1055/a-1001-5265

Die Hebamme 2019; 32: 7-16

(c) Georg Thieme Verlag KG Stuttgart · New York ISSN 0932-8122 